\title{
Clinical Applications of Ketogenic Diet-Induced Ketosis in Neurodegenerative and Metabolism- Related Pathologies ${ }^{+}$
}

\author{
Raffaele Pilla \\ External Pharmacy of Saint John of God-Fatebenefratelli Hospital, Viale Principe di Napoli 14/B, \\ 48100 Benevento, Italy; raf.pilla@gmail.com \\ + Presented at the The 1st International Electronic Conference on Nutrients - Nutritional and Microbiota \\ Effects on Chronic Disease, 2-15 November 2020; Available online: https://iecn2020.sciforum.net/. \\ Published: 30 October 2020
}

\begin{abstract}
Metabolic-based therapies such as nutritional ketosis have been proven effective for seizure disorders and various acute and chronic neurological pathologies. In a healthy brain, glucose is the primary metabolic fuel for cells. However, neurodegenerative disorders, such as Alzheimer's disease (AD), Parkinson's disease (PD), seizure disorders, and traumatic brain injury (TBI) are associated with impaired glucose transport and metabolism and with mitochondrial dysfunction leading to energy deficit. Therapeutic ketosis can be considered as a form of metabolic therapy by providing alternative energy substrates. In addition, ketosis leads to metabolic adaptations that improve brain metabolism, restore mitochondrial ATP production, decrease reactive oxygen species production, reduce inflammation, and increase the activity of neurotrophic factors. Moreover, the synaptic activity between neurons is also stabilized through the increase of Szent-Györgyi-Krebs cycle intermediates, antioxidant effects, increased GABA-to-glutamate ratio, and activation of ATP-sensitive potassium channels.
\end{abstract}

Keywords: Ketogenic; diet; ketogenic diet; ketosis; therapeutic ketosis; cancer; obesity; epilepsy; Glut 1 Deficit; Metabolic Syndrome

\section{Introduction}

The Ketogenic Diet (KD) represents a well-known therapeutic option for refractory epilepsy [1], although mechanisms regulating its anticonvulsant effects are currently object of study [2]. The body is constantly producing small amounts of ketone bodies that, under physiological conditions, can make 22 ATP each, and it is regulated mainly by insulin. In a metabolic state of ketosis, ketone body production is augmented when circulating carbohydrates decrease or fatty acids blood levels are raised [3].

Although the mechanisms underlying the anti-seizure effects of the high-fat ketogenic diet (KD) remain unclear, it has been long-questioned whether ketone bodies (i.e., $\beta$-hydroxybutyrate, acetoacetate and acetone) provide any contribution from a mechanistic perspective. In addition it has been investigated whether their effect is exploited in combination or alone [4]. It has been observed that ketone bodies are responsible for enhanced fatty acid oxidation and for a global shift toward intermediary metabolism. However, it has been shown that they are not pivotal for the diet's clinical effects [5]. This finding was proven by the fact that $\beta$-hydroxybutyrate blood concentrations did not correspond to seizure control observations [6]. Nevertheless, it has been observed that $\beta$ hydroxybutyrate may interact with multiple novel molecular targets such as histone deacetylases [7], hydroxycarboxylic acid receptors on immune cells [8], and the NLRP3 inflammasome [9]. 
Overall, as a diet-based therapy is expected to render a broad array of biochemical, molecular, and cellular changes, no single mechanism can explain how the KD works.

Among diets developed to address health problems, the ketogenic diet has had a long and variable history. It was originally developed in the early 1920s at John Hopkins' All Children Hospital in Baltimore, MD as an alternative strategy to help children with epilepsy. Later, as pharmacological approaches became available and effectively addressed seizures, the diet fell out of favor [10]. During the last few decades, researchers and clinicians have learned and proven that it can be useful in children and adults with refractory epilepsy and a variety of other pathological conditions. This very high-fat diet almost fully takes carbohydrates off of the patient's food selection. The result is the substitution of ketone bodies as a source of energy. Today's ketogenic diet has been modified with scientifically proven adjustments to increase palatability and help with compliance. It has been proven to be effective for some forms of epilepsy (mostly refractory), and has shown to have neuroprotective effects in Alzheimer's disease, Parkinson's disease, and glaucoma. In addition, many patients are using it to lose weight and/or prevent obesity [10].

Human brain derives over $60 \%$ of its energy from ketones when glucose availability is limited. After prolonged periods of fasting or during a KD, the whole body utilizes energy obtained from free fatty acids (FFAs) released from adipose tissue. However, FFAs are insufficient to provide consistent energy for the brain, this is why hepatic ketogenesis converts them into ketone bodies: $\beta$ hydroxybutyrate (BHB) and acetoacetate (AcAc), while a percentage of AcAc spontaneously decarboxylates to acetone [11]. Recent perspectives about the KD potentials and neuroprotective properties strongly support its experimental and clinical application in a wide plethora of different neurological diseases [12]. Notably, the metabolic state of mild ketosis, induced through KD administration, calorie restriction or fasting, may be used to metabolically manage epilepsy and a number neurodegenerative syndromes [13], amyotrophic lateral sclerosis [14], and some types of cancer $[15,16]$.

In addition, epigenetics has been playing an important role in the world of ketosis. In fact, it has been shown how dietary restriction may delay the aging process associated with histone modifications. In this scenario, KD represents a valid therapeutic tool in inflammatory nervous system-related neurodegenerative pathologies, such as Parkinson's Disease $[17,18]$.

According to the literature, the KD might exert its neuroprotective effect through an inflammatory cytokine and chemokine modulation with a resultant reduction of lymphocyte proliferation and an oxidative stress reduction [19]. In fact, the cytokine/chemokine modulation may prevent the activation of the inflammatory cascade with a consequent reduction in free radical production, also known as reactive oxygen species, ROS [20].

In addition, KD has shown an intrinsic antioxidant effect, considered the experimental observation in murine models for glutathione-peroxidase increased activity in the hippocampus while following a KD [21,22]. In this light, the evident antioxidant properties of the KD may provide a significant neuroprotective effect against a number of neurodegenerative syndromes [12,23].

Moreover, the KD has shown to have protective properties on the synaptic region of hippocampal sections undergone to metabolic deficit conditions induced by low glucose levels, correlated to up-regulation of genes coding for mitochondrial ATP-synthase [24].

Similar results have been observed in a relatively recent study, where ketone bodies have shown to provide protective effects on synapses after the mitochondrial respiratory chain inhibition, through an ATP production and antioxidant activity increase [25].

However, ketosis has also been observed to have detrimental effects on the nervous system. In fact, some relatively recent studies suggested that ketosis might accelerate the neurodegenerative process [26]. Furthermore, few works have been questioning about its side effects; notably, consultant pharmacists have addressed questions about the KD, its potential to correct or alleviate health conditions, and its limitations with an emphasis on the elderly [10].

In addition, it has been suggested that in many cases it is hard to obtain sufficient compliance from the patients. This is why it has been suggested that it might not be practical for most people to 
adhere to ketogenic diets continuously and alternative strategies are needed to harness the brainprotective potential of ketone bodies [27].

Furthermore, it has been observed that the KD can lead to an augmented expression and uncoupling protein activity (UCPs), which are proteins responsible for the mitochondrial transportation, which down-regulation seems to be associated to a higher susceptibility to the experimental autoimmune encephalomyelitis (EAE) activity [28], thus facilitating the inflammatory processes and the ROS production, leading to worse motor performances [29].

Taken together, the experimental data suggest the adoption of KD for PD patients in order to restore the bio-energetic balance with potential neuroprotective effects [30], also due to a consistent improvement in L-Dopa absorption [31]. In fact, it has been demonstrated in some animal models that one of the major metabolites, $\beta$-hydroxybutyrate, can reduce the substantia nigra neuron loss and increase the oxygen consumption in mitochondria [32,33]. The beneficial effects of KD on mitochondrial activity explains the improvement of patients' scores in Parkinson' disease [30]. In addition, it has been observed that cortical contusions may be decreased in an animal model of cortical injury through therapeutic ketosis [34]. Furthermore, the KD might improve the health status of patients following traumatic brain injury (TBI), as this clinical condition may lead to epilepsy in some cases [35].

Overall, ketone supplements are currently being developed, and also medical foods and dietary supplements are emerging in order to help keep low blood glucose levels and elevate ketone levels without forcing any dietary restrictions on patients, whom difficult clinical conditions might make hard to follow.

Some old studies [36-38] proved that the mild $\mathrm{H}^{+}$load and blood $\mathrm{pH}$ physiologically return back to normal ranges as long as ketones are maintained below the value of $10 \mathrm{mM}$ [39]. However, an important concern that arose was that blood $\mathrm{pH}$ may transiently decrease during the initial phases of ketosis, which is due to the accumulation of ketone bodies in the bloodstream [40]. This topic is still under discussion and one of the major aspects to consider is the common confusion about the physiological state of nutritional ketosis in the medical community: ketone bodies were previously considered as "toxic metabolites" [30], and thus usually caregivers associate the definition of "therapeutic ketosis" with "diabetic ketoacidosis", which is responsible for the well-known runaway ketosis and might lead to ketone bodies concentrations of $20 \mathrm{mM}$ or greater. It is pivotal to underline that the difference between the two metabolic states; in fact, ketone blood concentrations during therapeutic ketosis can vary between 0.5 and $8 \mathrm{mM}$ [41].

Conflicts of Interest: The authors whose names are listed immediately below certify that they have NO affiliations with or involvement in any organization or entity with any financial interest (such as honoraria, educational grants, participation in speakers' bureaus, membership, employment, consultancies, stock ownership, or other equity interest, and expert testimony or patent-licensing arrangements), or non-financial interest (such as personal or professional relationships, affiliations, knowledge or beliefs) in the subject matter or materials discussed in this manuscript.

\section{References}

1. Arya, R.; Peariso, K.; Gainza-Lein, M.; Harvey, J.; Bergin, A.; Brenton, J.N.; Burrows, B.T.; Glauser, T.; Goodkin, H.P.; Lai, Y.C.; et al. Efficacy and safety of ketogenic diet for treatment of pediatric convulsive refractory status epilepticus. Epilepsy Res. 2018, 144, 1-6.

2. French, J.A.; Koepp, M.; Naegelin, Y.; Vigevano, F.; Auvin, S.; Rho, J.M.; Rosenberg, E.; Devinsky, O.; Olofsson, P.S.; Dichter, M.A. Clinical studies and anti-inflammatory mechanisms of treatments. Epilepsia 2017, 58 (Suppl. 3), 69-82.

3. Dhillon, K.K.; Gupta, S. Biochemistry, Ketogenesis; StatPearls: Treasure Island, FL, USA, 2018.

4. Simeone, T.A.; Simeone, K.A.; Stafstrom, C.E.; Rho, J.M. Do ketone bodies mediate the anti-seizure effects of the ketogenic diet? Neuropharmacology 2018, 133, 233-241.

5. Simeone, T.A.; Simeone, K.A.; Rho, J.M. Ketone Bodies as Anti-Seizure Agents. Neurochem. Res. 2017, 42, 2011-2018.

6. Si, J.; Wang, S.; Liu, N.; Yang, X.; Wang, Y.; Li, L.; Wang, J.; Lv, X. Anticonvulsant effect of exogenous betahydroxybutyrate on kainic acid-induced epilepsy. Exp. Ther. Med. 2017, 14, 765-770. 
7. Zhang, J.; Li, X.; Ren, Y.; Zhao, Y.; Xing, A.; Jiang, C.; Chen, Y.; An, L. Intermittent Fasting Alleviates the Increase of Lipoprotein Lipase Expression in Brain of a Mouse Model of Alzheimer's Disease: Possibly Mediated by beta-hydroxybutyrate. Front. Cell. Neurosci. 2018, 12, 1.

8. Offermanns, S. Hydroxy-Carboxylic Acid Receptor Actions in Metabolism. Trends Endocrinol. Metab. 2017, 28, 227-236.

9. Goldberg, E.L.; Asher, J.L.; Molony, R.D.; Shaw, A.C.; Zeiss, C.J.; Wang, C.; Morozova-Roche, L.A.; Herzog, R.I.; Iwasaki, A.; Dixit, V.D. beta-Hydroxybutyrate Deactivates Neutrophil NLRP3 Inflammasome to Relieve Gout Flares. Cell Rep. 2017, 18, 2077-2087.

10. Walczyk, T.; Wick, J.Y. The Ketogenic Diet: Making a Comeback. Consult. Pharm. J. Am. Soc. Consult. Pharm. 2017, 32, 388-396.

11. Cahill, G.F., Jr. Fuel metabolism in starvation. Annu. Rev. Nutr. 2006, 26, 1-22.

12. Augustin, K.; Khabbush, A.; Williams, S.; Eaton, S.; Orford, M.; Cross, J.H.; Heales, S.J.R.; Walker, M.C.; Williams, R.S.B. Mechanisms of action for the medium-chain triglyceride ketogenic diet in neurological and metabolic disorders. Lancet. Neurol. 2018, 17, 84-93.

13. Hartman, A.L.; Stafstrom, C.E. Harnessing the power of metabolism for seizure prevention: Focus on dietary treatments. Epilepsy Behav. 2013, 26, 266-272.

14. Zhao, W.; Varghese, M.; Vempati, P.; Dzhun, A.; Cheng, A.; Wang, J.; Lange, D.; Bilski, A.; Faravelli, I.; Pasinetti, G.M. Caprylic triglyceride as a novel therapeutic approach to effectively improve the performance and attenuate the symptoms due to the motor neuron loss in ALS disease. PLoS ONE 2012, 7, e49191.

15. Seyfried, T.N.; Flores, R.E.; Poff, A.M.; D’Agostino, D.P. Cancer as a metabolic disease: Implications for novel therapeutics. Carcinogenesis 2014, 35, 515-527.

16. Poff, A.M.; Ward, N.; Seyfried, T.N.; Arnold, P.; D'Agostino, D.P. Non-Toxic Metabolic Management of Metastatic Cancer in VM Mice: Novel Combination of Ketogenic Diet, Ketone Supplementation, and Hyperbaric Oxygen Therapy. PLoS ONE 2015, 10, e0127407.

17. Paoli, A.; Bianco, A.; Damiani, E.; Bosco, G. Ketogenic diet in neuromuscular and neurodegenerative diseases. BioMed Res. Int. 2014, 2014, 474296.

18. Moreno, C.L.; Mobbs, C.V. Epigenetic mechanisms underlying lifespan and age-related effects of dietary restriction and the ketogenic diet. Mol. Cell. Endocrinol. 2017, 455, 33-40.

19. Achanta, L.B.; Rae, C.D. Beta-Hydroxybutyrate in the Brain: One Molecule, Multiple Mechanisms. Neurochem. Res. 2017, 42, 35-49.

20. Kim, D.Y.; Vallejo, J.; Rho, J.M. Ketones prevent synaptic dysfunction induced by mitochondrial respiratory complex inhibitors. J. Neurochem. 2010, 114, 130-141.

21. Kim, D.Y.; Hao, J.; Liu, R.; Turner, G.; Shi, F.D.; Rho, J.M. Inflammation-mediated memory dysfunction and effects of a ketogenic diet in a murine model of multiple sclerosis. PLoS ONE 2012, 7, e35476.

22. Kephart, W.C.; Mumford, P.W.; Mao, X.; Romero, M.A.; Hyatt, H.W.; Zhang, Y.; Mobley, C.B.; Quindry, J.C.; Young, K.C.; Beck, D.T.; et al. The 1-Week and 8-Month Effects of a Ketogenic Diet or Ketone Salt Supplementation on Multi-Organ Markers of Oxidative Stress and Mitochondrial Function in Rats. Nutrients 2017, 9, 1019.

23. van Horssen, J.; Schreibelt, G.; Drexhage, J.; Hazes, T.; Dijkstra, C.D.; van der Valk, P.; de Vries, H.E. Severe oxidative damage in multiple sclerosis lesions coincides with enhanced antioxidant enzyme expression. Free Radic. Biol. Med. 2008, 45, 1729-1737.

24. Bough, K.J.; Wetherington, J.; Hassel, B.; Pare, J.F.; Gawryluk, J.W.; Greene, J.G.; Shaw, R.; Smith, Y.; Geiger, J.D.; Dingledine, R.J. Mitochondrial biogenesis in the anticonvulsant mechanism of the ketogenic diet. Ann. Neurol. 2006, 60, 223-235.

25. Greco, T.; Glenn, T.C.; Hovda, D.A.; Prins, M.L. Ketogenic diet decreases oxidative stress and improves mitochondrial respiratory complex activity. J. Cereb. Blood Flow Metab. Off. J. Int. Soc. Cereb. Blood Flow Metab. 2016, 36, 1603-1613.

26. Lauritzen, K.H.; Hasan-Olive, M.M.; Regnell, C.E.; Kleppa, L.; Scheibye-Knudsen, M.; Gjedde, A.; Klungland, A.; Bohr, V.A.; Storm-Mathisen, J.; Bergersen, L.H. A ketogenic diet accelerates neurodegeneration in mice with induced mitochondrial DNA toxicity in the forebrain. Neurobiol. Aging 2016, 48, 34-47.

27. McCarty, M.F.; DiNicolantonio, J.J.; O'Keefe, J.H. Ketosis may promote brain macroautophagy by activating Sirt1 and hypoxia-inducible factor-1. Med. Hypotheses 2015, 85, 631-639. 
28. Choi, I.Y.; Piccio, L.; Childress, P.; Bollman, B.; Ghosh, A.; Brandhorst, S.; Suarez, J.; Michalsen, A.; Cross, A.H.; Morgan, T.E.; et al. A Diet Mimicking Fasting Promotes Regeneration and Reduces Autoimmunity and Multiple Sclerosis Symptoms. Cell Rep. 2016, 15, 2136-2146.

29. Vogler, S.; Pahnke, J.; Rousset, S.; Ricquier, D.; Moch, H.; Miroux, B.; Ibrahim, S.M. Uncoupling protein 2 has protective function during experimental autoimmune encephalomyelitis. Am. J. Pathol. 2006, 168, 15701575 .

30. Hashim, S.A.; VanItallie, T.B. Ketone body therapy: From the ketogenic diet to the oral administration of ketone ester. J. Lipid Res. 2014, 55, 1818-1826.

31. Jabre, M.G.; Bejjani, B.P. Treatment of Parkinson disease with diet-induced hyperketonemia: A feasibility study. Neurology 2006, 66, 617.

32. D'Agostino, D.P.; Pilla, R.; Held, H.E.; Landon, C.S.; Puchowicz, M.; Brunengraber, H.; Ari, C.; Arnold, P.; Dean, J.B. Therapeutic ketosis with ketone ester delays central nervous system oxygen toxicity seizures in rats. Am. J. Physiol. Regul. Integr. Comp. Physiol. 2013, 304, R829-R836.

33. Viggiano, A.; Pilla, R.; Arnold, P.; Monda, M.; D’Agostino, D.; Coppola, G. Anticonvulsant properties of an oral ketone ester in a pentylenetetrazole-model of seizure. Brain Res. 2015, 1618, 50-54.

34. Prins, M.L.; Fujima, L.S.; Hovda, D.A. Age-dependent reduction of cortical contusion volume by ketones after traumatic brain injury. J. Neurosci. Res. 2005, 82, 413-420.

35. McDougall, A.; Bayley, M.; Munce, S.E. The ketogenic diet as a treatment for traumatic brain injury: A scoping review. Brain Inj. 2018, 32, 416-422.

36. Desrochers, S.; Dubreuil, P.; Brunet, J.; Jette, M.; David, F.; Landau, B.R.; Brunengraber, H. Metabolism of (R,S)-1,3-butanediol acetoacetate esters, potential parenteral and enteral nutrients in conscious pigs. Am. J. Physiol. 1995, 268, E660-E667.

37. Ciraolo, S.T.; Previs, S.F.; Fernandez, C.A.; Agarwal, K.C.; David, F.; Koshy, J.; Lucas, D.; Tammaro, A.; Stevens, M.P.; Tserng, K.Y.; et al. Model of extreme hypoglycemia in dogs made ketotic with (R,S)-1,3butanediol acetoacetate esters. Am. J. Physiol. 1995, 269, E67-E75.

38. Puchowicz, M.A.; Smith, C.L.; Bomont, C.; Koshy, J.; David, F.; Brunengraber, H. Dog model of therapeutic ketosis induced by oral administration of R,S-1,3-butanediol diacetoacetate. J. Nutr. Biochem 2000, 11, 281287.

39. Withrow, C.D. The ketogenic diet: Mechanism of anticonvulsant action. Adv. Neurol. 1980, 27, $635-642$.

40. Stubbs, B.J.; Cox, P.J.; Evans, R.D.; Santer, P.; Miller, J.J.; Faull, O.K.; Magor-Elliott, S.; Hiyama, S.; Stirling, M.; Clarke, K. On the Metabolism of Exogenous Ketones in Humans. Front. Physiol. 2017, 8, 848.

41. Van der Auwera, I.; Wera, S.; Van Leuven, F.; Henderson, S.T. A ketogenic diet reduces amyloid beta 40 and 42 in a mouse model of Alzheimer's disease. Nutr. Metab. (Lond.) 2005, 2, 28.

Publisher's Note: MDPI stays neutral with regard to jurisdictional claims in published maps and institutional affiliations.

(C) 2020 by the authors. Submitted for possible open access publication under the terms and conditions of the Creative Commons Attribution (CC BY) license (http://creativecommons.org/licenses/by/4.0/). 